\title{
SLC6A3 Polymorphism Predisposes to Dopamine Overdose in Parkinson's Disease
}

\begin{abstract}
Brian D. Robertson ${ }^{1}$, Abdullah S. Al Jaja ${ }^{2,3}$, Alex A. MacDonald ${ }^{4}$, Nole M. Hiebert ${ }^{2,5}$, Ruzbeh Tamjeedi ${ }^{6}$, Ken N. Seergobin ${ }^{2}$, Ute I. Schwarz ${ }^{5,7}$, Richard B. Kim ${ }^{5,7}$ and Penny A. MacDonald $2,3,5,8 *$

${ }^{1}$ Schulich School of Medicine \& Dentistry, University of Western Ontario, London, ON, Canada, ${ }^{2}$ Brain and Mind Institute, University of Western Ontario, London, ON, Canada, ${ }^{3}$ Department of Neuroscience, University of Western Ontario, London, ON, Canada, ${ }^{4}$ Department of Medicine, Undergraduate Faculty of Medicine, University of Toronto, Toronto, ON, Canada, ${ }^{5}$ Department of Physiology and Pharmacology, University of Western Ontario, London, ON, Canada, ${ }^{6}$ Faculty of Law, University of Ottawa, Ottawa, ON, Canada, ' Division of Clinical Pharmacology, Department of Medicine, Schulich School of Medicine \& Dentistry, University of Western Ontario, London, ON, Canada, ${ }^{8}$ Department of Clinical Neurological Sciences, Schulich School of Medicine \& Dentistry, University of Western Ontario, London, ON, Canada
\end{abstract}

\section{OPEN ACCESS}

Edited by: Antonio Pisani,

Università degli Studi di Roma Tor Vergata, Italy

Reviewed by: Erwan Bezard,

UMR5293 Institut des Maladies Neurodégénératives (IMN), France Salvatore Galati, Neurocenter of Southern Switzerland (NSI), Switzerland

*Correspondence:

Penny A. MacDonald penny.macdonald@gmail.com

Specialty section: This article was submitted to Movement Disorders, a section of the journal Frontiers in Neurology

Received: 02 May 2018 Accepted: 31 July 2018 Published: 21 August 2018

Citation:

Robertson BD, Al Jaja AS, MacDonald AA, Hiebert NM, Tamjeedi R, Seergobin KN,

Schwarz UI, Kim RB and MacDonald PA (2018) SLC6A3 Polymorphism Predisposes to Dopamine Overdose in Parkinson's Disease. Front. Neurol. 9:693. doi: 10.3389/fneur.2018.00693
In Parkinson's disease (PD), cognitive functions mediated by brain regions innervated by ventral tegmental area (VTA) worsen with dopamine replacement therapy, whereas processes relying on regions innervated by the substantia nigra pars compacta (SNc) improve. The SLC6A3 gene encodes the dopamine transporter (DAT). The common 9R polymorphism produces higher DAT concentrations and consequently lower baseline dopamine than SLC6A3 wildtype. Whether SLC6A3 genotype modulates the effect of dopaminergic therapy on cognition in PD is not known. We investigated the effect of dopaminergic therapy and SLC6A3 genotype on encoding and recall of abstract images using the Aggie Figures Learning Test in PD patients. Encoding depends upon brain regions innervated by the VTA, whereas recall is mediated by widespread brain regions, a number innervated by the SNc. We found that dopaminergic therapy worsened encoding of abstract images in 9R carriers only. In contrast, dopaminergic therapy improved recall of abstract images in all PD patients, irrespective of SLC6A3 genotype. Our findings suggest that 9R-carrier PD patients are more predisposed to dopamine overdose and medication-induced impairment of cognitive functions mediated by VTA-innervated brain regions. Interestingly, PD patients without the 9R polymorphism did not show such an impairment. SLC6A3 genotype does not modulate the dopaminergic therapy-induced improvement of functions mediated by SNc-innervated regions in PD patients.

Keywords: Parkinson's disease, polymorphism, SLC6A3, overdose, dopamine, encoding

\section{INTRODUCTION}

Parkinson's disease (PD) is a neurodegenerative disorder characterized by substantial dopamineproducing neuron loss in the substantia nigra pars compacta $(\mathrm{SNc})$ with relative sparing of dopamine-producing neurons in the ventral tegmental area (VTA). The SNc principally supplies dopamine to the dorsal striatum (DS), comprising the bulk of the putamina and caudate nuclei. The ensuing depletion of dopamine to the DS produces the cardinal PD motor manifestations of 
rigidity, tremor, and bradykinesia (1). Dopamine replacement medication reliably and effectively improves these DS-mediated motor symptoms in PD (2).

Although motor symptoms are uniformly improved by dopaminergic therapy in $\mathrm{PD}$, distinct cognitive functions are dissimilarly affected by dopamine replacement therapy. Some cognitive functions are worsened whereas others are ameliorated or redressed by dopaminergic medication (2). Functions that depend upon brain regions receiving dopamine from the relatively-spared VTA have been found quite consistently to be worsened by exogenous dopamine therapy (2). The detrimental effects of dopaminergic therapy on cognition have been attributed to an overdose of dopamine in VTA-innervated brain regions that receive normal or near-normal dopamine in PD (2-5). These VTA-innervated regions include the ventral striatum (VS)-comprising the nucleus accumbens and most ventral parts of putamen and caudate nuclei, orbitofrontal cortex, prefrontal and limbic cortical regions, including the hippocampus (2-5). In contrast, there is now an ample literature suggesting that cognitive functions that depend upon DS, or cortical regions reciprocally connected to DS, are improved by dopaminergic therapy $(2-5)$.

Of importance to the current study, learning, in its various forms, is mediated by VTA-innervated regions such as the VS, hippocampus, and medial frontal cortex (6-9). This function is normal at baseline and worsened by dopaminergic medications in PD $(2,10-13)$ and in healthy adults $(14,15)$. On the other hand, decision making and memory retrieval implicate DS. These functions are impaired at baseline and improve with dopaminergic therapy in $\mathrm{PD}(2,4,16-18)$.

Dopamine transporter (DAT), encoded by gene SLC6A3, is a membrane transporter protein that resorbs synaptic dopamine. SLC6A3 is abundant in the striatum, midbrain, and hippocampus, but scarce in the prefrontal cortex (19)where synaptic dopamine is degraded primarily by catechol$O$-methyltransferase (COMT). In the SLC6A3 gene, a 40-base pair variable nucleotide tandem repeat element exists, with 9(9R) and 10-repeat (10R) forms being most prevalent (20). Recent meta-analyses $(21,22)$ that analyzed data from studies in which positron emission tomography and single photon emission computed tomography was used have clarified that presence of the SLC6A3 9R allele causes higher DAT levels than 10R-homozygosity. Hence, 9R-carriers are expected to have lower baseline dopamine concentrations compared to 10Rhomozygotes.

In 9R carriers, increased expression of DAT, and consequently lower concentrations of dopamine at baseline, are expected to enhance the ratio between phasic, pulsatile, dopamine bursts related to events such as reward, positive feedback, or behavior, and tonic, basal dopamine release that occurs at rest. This enhanced signal-to-noise ratio is expected to result in more efficient signaling and potentially improved learning and performance. However, given that this superior performance is expected on the basis of lower tonic, basal dopamine, 9R carriers are predicted to be more susceptible to overdose effects of exogenous dopamine.
In line with the hypothesis that $9 \mathrm{R}$ carriers have more efficient dopamine signaling and potentially improved performance, $9 \mathrm{R}$ carriers have been shown to have enhanced activity in bilateral striatum upon the reception of positive feedback $(23,24)$. Dreher et al. also found that $9 \mathrm{R}$ carriers had greater reactivity in the midbrain and lateral PFC upon the reception of reward and, further, showed enhanced reactivity in DS and VS during reward anticipation (25). Further, in a PET study of habitual smokers, increased smoking-related VS reactivity-hypothesized to be due to larger phasic dopamine bursts in $9 \mathrm{R}$ carriers, who have lower tonic synaptic dopamine concentrations-was seen in $9 \mathrm{R}$ carriers relative to $10 \mathrm{R} / 10 \mathrm{R}$ homozygotes (26). $9 \mathrm{R}$ carriers, as compared to $10 \mathrm{R} / 10 \mathrm{R}$ homozygotes, have also been shown to evidence a larger frontoparietal, novelty-dependent electroencephalographic response during the presentation of auditory cues signaling a task switch during a test of cognitive flexibility (27). These results suggest that $9 \mathrm{R}$ carriers are more sensitive to phasic increases in dopamine in the striatum.

The effect of $S L C 6 A 3$ gene polymorphisms on cognition in PD patients has scarcely been investigated (28). In the lone investigation of this SLC6A3 polymorphism in PD using neuroimaging, patients carrying a $9 \mathrm{R}$ allele exhibited less activation than their 10R homozygous counterparts in caudate nucleus and prefrontal and premotor cortices when planning and executing a set-shift (28). This is in contradistinction to studies with healthy controls in which 9R-carriers consistently exhibit greater cognition-related neural activation relative to 10R-homozygotes using neuroimaging $(24,25,29,30)$.

To our knowledge, how SLC6A3 polymorphisms affect response to dopaminergic therapy has not been examined in PD. In healthy controls, dopaminergic therapy reduced abilities of healthy 9R-carriers relative to 10R-homozygotes to maximize earnings by learning and adapting to the playing style of their opponents in a competitive task (31). In contrast, dopaminergic therapy improved cognitive flexibility, a DS-mediated function (2, 32 ), in healthy 10R-homozygotes but not 9R-carriers (27). These findings present the intriguing possibility that SLC6A3 genotype interacts with the now well-described differential medication effects in VTA-innervated brain regions vs. DS $(2,3)$.

\section{Current Study}

This study was designed to investigate the role of the SLC6A3 polymorphism on memory encoding and retrieval in PD patients on and off dopaminergic medication. Identifying genemedication interactions for cognitive symptoms in PD patients would be important from both a clinical and basic science standpoint. Clinically, identifying genes that interact with medication to differentially affect cognition could lead to more customized treatment regimens to optimize function and limit side effects. From a basic science standpoint, this study could yield valuable insights into the mechanisms of memory encoding and retrieval, taking into account variation in endogenous and exogenous dopamine signaling.

We have previously shown that dopaminergic therapy worsens encoding and improves recall of abstract images in PD (11). This pattern of results is consistent with literature ascribing encoding to VTA-innervated brain regions such as 
hippocampus and VS, and recall to brain regions including DS $(6,7,33)$, which are differentially dopamine depleted in PD (1). Here, we implemented this encoding and retrieval methodology to investigate whether SLC6A3 gene polymorphisms impact cognition and responses to dopaminergic therapy in $\mathrm{PD}$, and particularly whether these effects are dissimilar for functions mediated by VTA-innervated brain regions vs. DS.

Overall, for PD patients off medication, we expected to see $9 \mathrm{R}$ participants outperform 10R/10R participants. On medication, we further predicted that $9 \mathrm{R} P D$ patients would be more sensitive to overdose of VTA-innervated brain regions from exogenous dopamine. Hence, 9R carriers were expected to have greatest impairment in memory encoding on relative to off dopaminergic therapy. For recall, mediated by DS and its cortical partners, we expected that all PD patients would recall more items in the $\mathrm{ON}$ than OFF dopaminergic state.

\section{METHODS}

\section{Participants}

Forty-five patients with PD participated in this study. Patients were diagnosed by a licensed neurologist and met the core assessment criteria for diagnosis of idiopathic PD for surgical interventional therapy and the UK Brain Bank criteria for PD. All patients who participated in this study were referred directly from licensed neurologists. Participants were excluded if they were previously diagnosed with dementia or mild cognitive impairment, if they reported loss of a previous level of function related to cognitive problems, or if they scored less than 22/30 on the Montreal Cognitive Assessment (MOCA). Further, participants were excluded if they were abusing alcohol, prescription or street drugs, or taking medications such as donepezil, galantamine, rivastigmine, memantine, or methylphenidate. Participants were also excluded if they were known to have greater than mild-moderate depression (Beck Depression Inventory score above 30/63) or greater than mildmoderate anxiety (Beck Anxiety Inventory score above 30/63). They were also excluded if they had any other neurological illness. This study was carried out in accordance with the recommendations, and was approved by the ethics review board of both Health Sciences North (Sudbury, Ontario, Canada), and the University of Western Ontario (London, Ontario, Canada). All participants provided written, informed consent in accordance with the Declaration of Helsinki.

Presence as well as severity of disease were assessed for all patients both on and off dopaminergic medication using the motor subscale of the Unified Parkinson's Disease Rating Scale (UPDRS) by a licensed movement disorders neurologist (PAM). All patients were treated with dopamine replacement medications such as dopamine precursors, L-3,4dihydroxyphenylalanine (L-DOPA), and/or dopamine agonists. Table 1 presents mean group demographic information, screening affective and cognitive measures, and daily doses of DA-replacement medications in L-DOPA equivalents (LED). Calculation of daily LED for each patient was based on theoretical equivalence to L-DOPA (34) as follows: L-DOPA dose + L-DOPA dose $\mathrm{x} 1 / 3$ if on entacapone + bromocriptine $(\mathrm{mg}) \times$
TABLE 1 | Demographic and screening data for PD patients and controls separated by genotype.

\begin{tabular}{|c|c|c|c|}
\hline & 10R/10R & 9R & $p$-value \\
\hline$N$ & 30 & 14 & \\
\hline Age & 68.07 (1.35) & $69.14(1.80)$ & ns \\
\hline Education & $15.13(0.50)$ & $14.35(0.86)$ & $n s$ \\
\hline Years disease & $6.83(1.29)$ & $6.27(1.30)$ & ns \\
\hline LED (mg) & 683.60 (62.62) & 687.63 (86.87) & ns \\
\hline L-DOPA $(n)$ & 29 & 14 & \\
\hline $\mathrm{DA}(n)$ & 13 & 5 & \\
\hline UPDRS (ON) & $16.83(1.00)$ & $16.82(0.86)$ & ns \\
\hline UPDRS (OFF) & $24.33(2.10)$ & $22.18(2.47)$ & $n s$ \\
\hline COMT Val/Nal (n) & 8 & 3 & \\
\hline COMT Val/Met $(n)$ & 16 & 10 & \\
\hline COMT Met/Met $(n)$ & 6 & 1 & \\
\hline BDI-II (ON) & $8.00(1.12)$ & $10.43(1.34)$ & $n s$ \\
\hline BDI-II (OFF) & $8.43(0.99)$ & $10.93(1.51)$ & $n s$ \\
\hline $\mathrm{BAl}(\mathrm{ON})$ & $8.60(1.23)$ & $12.00(2.63)$ & $n s$ \\
\hline $\mathrm{BAI}(\mathrm{OFF})$ & $10.00(1.48)$ & $11.50(1.92)$ & $n s$ \\
\hline SAS (ON) & $12.00(0.98)$ & $12.50(1.42)$ & $n s$ \\
\hline SAS (OFF) & $11.37(1.11)$ & $11.50(1.32)$ & $n s$ \\
\hline ANART IQ & $122.25(1.50)$ & 123.917 (2.40) & ns \\
\hline F-Words & $13.40(0.74)$ & $16.62(1.83)$ & $n s$ \\
\hline A-Words & $10.00(0.79)$ & $13.46(1.66)$ & 0.04 \\
\hline S-Words & $13.30(0.87)$ & $17.92(1.99)$ & 0.02 \\
\hline Animals & $19.03(1.08)$ & $19.69(1.74)$ & ns \\
\hline MOCA & $25.87(0.42)$ & $26.86(0.61)$ & ns \\
\hline
\end{tabular}

All values reported are group means (SEM). Education refers to the number of years spent in the education system. Elaboration of measures used in table follow below.

Education, years of education; Years disease, years since diagnosis of PD; L-DOPA, L3,4-dihydroxyphenylalanine; $L E D$, daily L-DOPA equivalent dose in mg; L-DOPA, number of $P D$ patients who were taking $L-D O P A, D A$, number of $P D$ patients who were taking dopamine agonist drugs; UPDRS, Unified Parkinson's Disease Rating Scale; BDI-II, Beck Depression Inventory II; BAl, Beck Anxiety Inventory; SAS, Starkstein Apathy Scale; ANART IQ, National Adult Reading Test IQ estimation (tested in the ON session only); $F_{-}, A_{-}$, or $S$-Words, number of words beginning with the letter $F_{\text {, }} A$, or $S$, respectively, generated in $60 \mathrm{~s}$ (tested in the ON session only); MOCA, total score on the Montreal Cognitive Assessment. Verbal fluency and MOCA tests were completed while on medication.

$10+$ cabergoline or pramipexole $(\mathrm{mg}) \times 67+$ ropinirole $(\mathrm{mg})$ $\times 20+$ pergolide $(\mathrm{mg}) \times 100+$ apomorphine $(\mathrm{mg}) \times 8$.

\section{Genotyping Procedure}

Saliva samples were collected from participants using Oragene $2 \mathrm{~mL}$ DNA collection kits (DNA Genotek, Ottawa, Ontario, Canada), and genomic DNA extracted using the MagNA Pure Compact instrument (Roche Diagnostics, Laval, Quebec, Canada). Genotyping of a 40-base pair (bp) variable nucleotide tandem repeat (VNTR; rs28363170) located in the 3 '-untranslated region of the SLC6A3 gene was carried out according to a previously described method $(35,36)$ with modifications. In brief, a polymerase chain reaction (PCR) was performed (Forward primer: 5'-TGT GGT GTA GGG AAC GGC CTG AG-3', reverse primer: 5'-CTT CCT GGA GGT CAC GGC TCA AGG-3') with the following conditions: initial cycle 
at $94^{\circ} \mathrm{C}$ for $5 \mathrm{~min}, 35 \mathrm{cycles}$ at $94^{\circ} \mathrm{C}$ for $30 \mathrm{~s}, 66^{\circ} \mathrm{C}$ for $30 \mathrm{~s}$, and $72^{\circ} \mathrm{C}$ for $1 \mathrm{~min}$, and final cycle at $72^{\circ} \mathrm{C}$ for $7 \mathrm{~min}$. PCR products were loaded on a $6 \% \mathrm{TBE}$ gel (Invitrogen) to separate a 440 -bp and a 480-bp amplicon representing the $9 \mathrm{R}$ and $10 \mathrm{R}$ alleles, respectively.

Genotyping for the COMT c.472G $>$ A (Val158Met; rs4680) polymorphism was performed using a TaqMan allelic discrimination assay (C_25746809_50; Applied Biosystems ${ }^{\circledR}$, Foster City, CA, EUA) using 50 ng of genomic DNA per reaction.

One participant was excluded due to an inability to properly determine his/her genotype for the SLC6A3 gene. As such, 44 PD patients were included in our subsequent analyses.

\section{Design and Procedure}

All participants performed two versions of the Aggie Figures Learning Test (AFLT) on two consecutive days (11, 37) (Figure 1). PD patients completed the AFLT once while on their usual dopamine-replacement therapy as prescribed by their treating neurologist (i.e., the $\mathrm{ON}$ state) and once while they were withdrawn from their dopamine-replacement therapy (i.e., the OFF state). We counterbalanced the ON-OFF order such that half the participants first completed the task while ON and the other half first completed the task while OFF their dopaminergic therapy. In the OFF Session, patients were instructed to abstain from taking L-DOPA for a minimum of 12 to a maximum of $18 \mathrm{~h}$, and dopamine agonists for a minimum of 16 to a maximum of $20 \mathrm{~h}$ before testing (Figure 1).

In each session, a set of 15 abstract symbols, List A, was presented to participants. These symbols were presented one at a time for $1,000 \mathrm{~ms}$ in the center of a computer monitor. Participants were instructed to try to remember as many of these symbols as possible. After the entire list had been presented, the participant was given $120 \mathrm{~s}$ to draw all the symbols that they could remember onto a piece of paper. This study-immediate recall procedure for List A was repeated five times in each version of the AFLT task (Figure 1).

A second set of 15 abstract symbols, List B, was then presented using parameters identical to those above, but List B was only presented a single time. Participants were then given $120 \mathrm{~s}$ to draw all the symbols they could remember after the presentation List B. Next, participants were asked to draw all the symbols that they could recall from List A again (Figure 1).

After a 30-min period of delay, during which participants performed distractor tasks (i.e., a number comparison task not reported here), participants were asked to draw all the symbols that they could freely recall from Lists A and B (Figure 1).

\section{Data Analysis}

The AFLT was scored by two researchers who were blinded to the identity of the participants (i.e., 10R/10R or 9R) and session (i.e., ON or OFF state). A single point was awarded for each recalled item that could be unambiguously identified. Therefore, items were classified as correct if they had minor distortions in their shape or orientation. Any discrepancies in scoring between the two scorers were addressed such that an agreement was reached concerning scoring of these items.

\section{Measure of Encoding}

The difference in the number of correctly recalled items from the first and final study-immediate recall phases was used as our metric of memory encoding (38). That is, the number of items successfully recalled in the first study-immediate recall phase was subtracted from the number of items successfully recalled in the final study-immediate recall phase. This was to control for the effects of working memory and recall abilities. This subtraction serves to better isolate the memory encoding performance. This strategy aims to eliminate effects related to working memory and retrieval abilities on performance, as working memory and retrieval demands are expected to contribute to performance equally for the first and the last study-immediate recall phases, with differences across phases owing more to a participants' ability to encode abstract images into long-term memory (38).

\section{Measure of Retrieval}

We used the total number of items recalled from List A after the 30-min delay divided by the total score achieved in the final study-immediate recall phase as our measure of memory retrieval, referred to as Weighted Recall. Unlike study-immediate recall phases, recall after delay is believed to preferentially index retrieval processes (39). Further, by correcting for the number of items recalled on the final study-immediate recall phase, retrieval can be assessed in a less biased manner, controlling for differences between individuals in encoding ability.

\section{Analyses}

Encoding scores and Weighted Recall scores were used as dependent measures in separate $2 \times 2$ mixed-design analyses of variance (ANOVAs) with Genotype (SLC6A3 10R/10R vs. 9R-carriers) as the between-subject variable, and Session (ON vs. OFF) as the within-subject variable. Where warranted by significant interaction results, we followed up with subsequent one-way ANOVAs with Session (ON vs. OFF) as the withinsubject factor to explore the simple effects of Session within Genotype.

\section{RESULTS}

\section{Demographic and Clinical Data}

When examining the effect of SLC6A3 genotype on our demographic, clinical, and screening measures, we only found significant differences on two of our four measures of verbal fluency. Here, we found that 9R PD patients produced more words beginning with the letters $A$ and $S$ in $60 \mathrm{~s}$ than their 10R/10R counterparts. Of note, our tests of verbal fluency were completed while patients were on medication. All other demographic, clinical, and screening measures did not differ between groups (Table 1). It was important to note that our groups did not differ in baseline UPDRS (our measure of disease severity), UPDRS in the ON state (our measure of therapeutic response), disease duration, or LED (our dopaminergic medication dosage). Non-significant differences with respect to disease duration and LED were confirmed with Bayesian analysis in which the Bayes Factors strongly supported the null hypothesis in each case (Disease duration: $\mathrm{BF}_{10}=0.329$; 

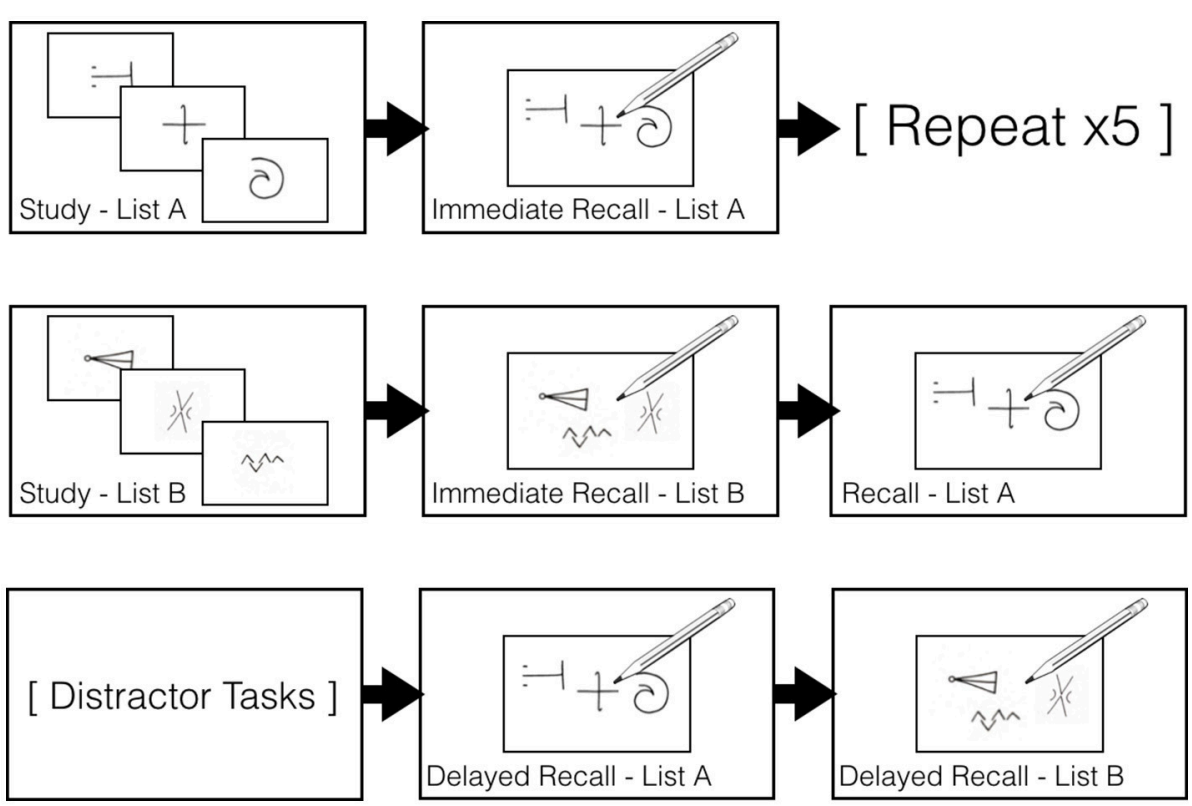

FIGURE 1 | Aggie Figures Learning Test design. First Row: The 15 items comprising List A were displayed, one-at-a-time, for 1,000 ms each on a computer screen. After all items had been displayed, participants were given $120 \mathrm{~s}$ to draw as many List A items as they could recall. This procedure was repeated five times in total. Second Row: The procedure above was completed a single time using the items from List B. Afterward, participants were then given 120 s to draw as many of the List A items they could recall. Third Row: Distractor tasks were completed for 30 mins. Participants were then asked to draw as many List A items they could recall. They were then asked to draw as many List B items as they could recall.

LED: $\left.\mathrm{BF}_{10}=0.337\right)$, suggesting that failure to find differences was not the result of lack of power.

\section{Genotyping Results}

Thirty participants were homozygous for the 10R allele and 14 participants were carriers of a single $9 \mathrm{R}$ allele and a 10R allele. No participants were homozygous for the $9 \mathrm{R}$ allele. The SLC6A3 gene distribution did not deviate from Hardy-Weinberg equilibrium $\left(\chi^{2}\right.$-test, $\left.p=1.00\right)$.

Additionally, due to the well-documented effects of the COMT rs4680 polymorphism on frontal dopamine (40), we also accounted for its distribution in our participants. There was no deviation from Hardy Weinberg equilibrium in the COMT rs4680 gene distribution $\left(\chi^{2}=1.62 ; p=0.20\right)$.

\section{Measure of Encoding}

We examined encoding scores in the AFLT in a $2 \times 2$ mixed ANOVA with SLC6A3 genotype (SLC6A3 10R/10R vs. 9Rcarriers) as the between-subject variable, and Session (ON vs. $\mathrm{OFF}$ ) as the within-subject variable (Table 2; Figure 2). There was no main effect of Session. We found that the Session x SLC6A3 interaction was significant $\left[F_{(1,42)}=4.840, M S E\right.$ $=13.182, p=0.033$, however. To better understand this interaction, we next examined Session effects for each of the SLC6A3 genotypes separately. For 9R carrier participants, there was a significant main effect of Session $\left[F_{(1,13)}=6.250, M S E=\right.$ $14.286, p=0.027]$. For $10 \mathrm{R} / 10 \mathrm{R}$ participants, there no main effect of Session $(F<1)$. In sum, we see worsening of memory encoding
TABLE 2 | Final study-immediate recall, encoding scores, and weighted recall scores for PD patients separated by SLC6A3 genotype.

\begin{tabular}{llccccc}
\hline & \multicolumn{2}{c}{ Encoding } & \multicolumn{2}{c}{ Weighted recall } \\
\hline & $\boldsymbol{n}$ & OFF & ON & OFF & ON \\
10R/10R & 30 & $4.83(0.45)$ & $5.07(0.43)$ & $0.99(0.05)$ & $1.27(0.75)$ \\
$9 \mathrm{R}$ & 14 & $5.86(0.75)$ & $4.43(0.55)$ & $1.03(0.09)$ & $1.22(0.15)$ \\
\hline
\end{tabular}

All values reported are group means (SEM). First trial values correspond to the mean number of items recalled by each group in the first study-immediate recall trial. Final recall values correspond to the mean number of items recalled by each group in the final studyimmediate recall trial. Encoding scores were calculated for each participant by subtracting the first recall score from the final recall score. Weighted recall scores were calculated by dividing the number of items recalled after a 30-min delay by the number of items recalled during the final study-immediate recall trial. 10R/10R groups are composed of PD patients who were homozygous for the 10R SLC6A3 40-bp VNTR allele. 9R groups are composed of PD patients who were heterozygous for the 9R SLC6A3 40-bp VNTR allele.

in the ON state (i.e., dopamine overdose effects) in the 9R carrier group only.

\section{Measure of Retrieval}

We examined Weighted Recall scores in the AFLT in a $2 \times 2$ mixed ANOVA with SLC6A3 genotype (SLC6A3 $10 \mathrm{R} / 10 \mathrm{R}$ vs. 9R-carriers) as the between-subject factor and Session (ON vs. OFF) as the within-subject variable (Table 2; Figure 3). We found a significant main effect of Session $\left[F_{(1,42)}=4.515, M S E=1.099, p=0.040\right]$, reflecting better recall performance when on relative to off dopaminergic medication. The Session x SLC6A3 interaction was not significant $(F<1)$. In summary, we found that the administration of dopamine 


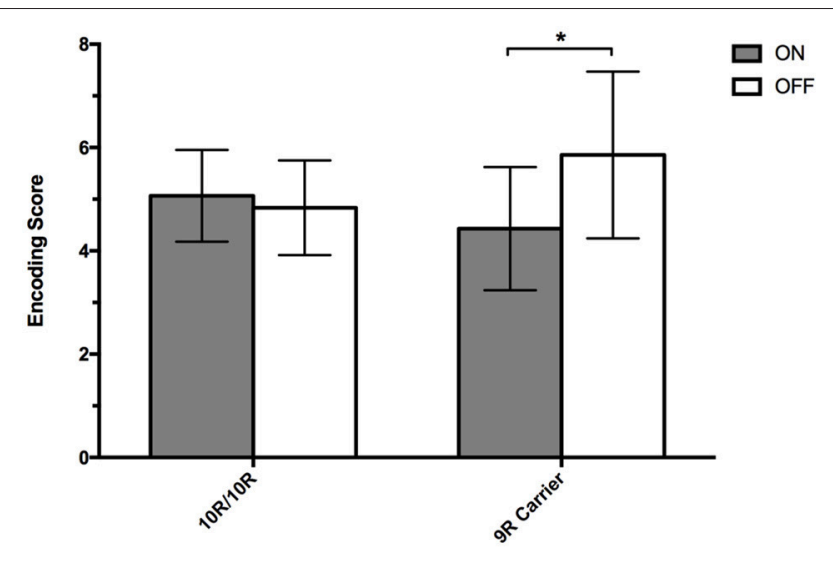

FIGURE 2 | Encoding Scores. Mean encoding scores ( \pm SEM) during the AFLT for PD patients, on and off dopaminergic medication, separated into 9R-carriers $(n=14)$ and 10R/10R-homozygotes $(n=30)$ of the SLC6A3 40-bp VNTR polymorphism. Mean encoding scores were calculated by subtracting the number of figures recalled in immediate-recall trial 1 from the number of figures recalled in immediate-recall trial 5 . A single asterisk represents $p=0.027$.

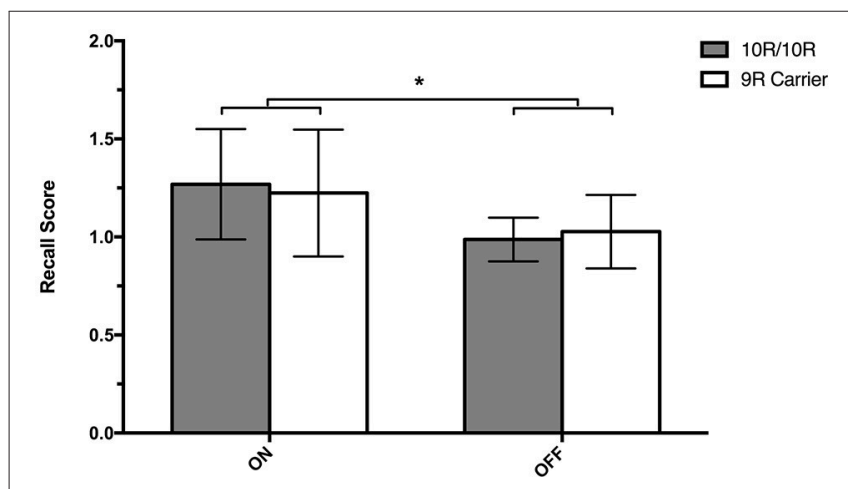

FIGURE 3 | Recall Scores. Mean recall scores ( \pm SEM) during the AFLT for PD patients, on and off medication, separated into 9R-carriers $(n=14)$ and 10R/10R-homozygotes $(n=30)$ of the DAT 40-bp VNTR polymorphism. Mean recall scores were calculated by dividing the number of figures recalled after the 30-min delay by the number of figures recalled during immediate-recall trial 5. A single asterisk represents $p=0.040$.

replacement medication improved recall scores in all PD patients. There were no differential effects related to SLC6A3 genotype.

\section{DISCUSSION}

\section{Summary of Results}

In this study, we investigated whether SLC6A3 genotype impacted cognition, response to dopaminergic therapy, or both in PD. In PD patients both on and off medication, we differentially assessed encoding of abstract images-a cognitive function mediated by VTA-innervated brain regions $(6,7,9$, 33) - and retrieval of these images - a cognitive function that implicates DS (41)-using the AFLT. We found no main effects of genotype on encoding, but did find a significant interaction between genotype and medication status. In 9Rcarriers, dopaminergic therapy worsened encoding scores, relative to performance in the OFF state. No ON-OFF effect was noted in $10 \mathrm{R}$-homozygotes. In this way, the $9 \mathrm{R}$ polymorphism appears to predispose to dopamine overdose. We found no main effect of genotype on retrieval, and genotype did not interact with medication status. However, we found that the administration of dopaminergic medication enhanced memory retrieval in PD patients overall.

Interpretation of Memory Encoding Results Encoding is consistently shown to recruit $(6,7,33)$ and depend upon $(42,43)$ VTA-innervated brain regions. A large and long-standing literature attributes explicit memory encoding to the VTA-innervated hippocampus (37). VS, another VTAinnervated brain region, is also implicated in memory encoding $(6,7,11,41,42)$, whether encoding is explicit and intentional (9) or implicit and unintentional (41), even when no reward, punishment, or feedback are present $(17,44,45)$.

Compared to the substantially-degenerated SNc, dopamineproducing neurons in the VTA are relatively spared in PD (1). Exogenous dopamine is administered to improve movement abnormalities by remediating dopamine deficiency in the SNc-innervated DS. However, dopaminergic therapy overdoses dopamine-replete VTA-innervated brain regions, such as VS, limbic, and prefrontal cortical regions, impairing their function in $\operatorname{PD}(2,3,12,13)$ and in healthy adults $(14,15)$.

In keeping with these pathophysiological details, whereas PD patients off dopaminergic medication perform comparably to healthy, age-matched controls, $\mathrm{PD}$ patients on dopaminergic therapy evidence impaired memory encoding $(10,11,46,47)$. In line with these investigations, we found that dopaminergic therapy worsened memory encoding in $\mathrm{PD}$ patients, but only for the carriers of the 9R polymorphism in SLC6A3 gene. These findings present the intriguing possibility that the 9R SLC6A3 gene predisposes to dopamine overdose in VTA-innervated brain regions.

\section{SLC6A3 Effects on Encoding On and Off Dopaminergic Therapy in PD}

There were no main effects of SLC6A3 genotype on encoding performance in our study, even though we had predicted superior encoding for PD 9R-carriers relative to 10R homozygotes. As predicted, however, we found that dopaminergic therapy produced greater impairment in encoding for 9R-carriers. In fact, we found no dopaminergic therapy-induced worsening of abstract figure learning in the 10R/10R group. This confirmed our expectations that $9 \mathrm{R}$-carriers are more sensitive to dopamine overdose. Of importance, there were no differences across genotype group in terms of severity of PD (UPDRS OFF), therapeutic response (UPDRS ON-OFF), PD duration, or dopaminergic dosage as expressed by LED to explain differential effect of dopaminergic therapy on encoding.

SLC6A3 affects re-uptake of dopamine, particularly in striatum and hippocampus. Recent meta-analyses have concluded that expression of SLC6A3 9R allele is higher than the 10R-homozygotes $(21,22)$. As such, 9R-carriers were predicted to have lower dopamine concentrations at baseline due 
to greater re-uptake. Lower baseline dopamine concentrations arguably yield a higher signal-to-noise ratio, with more impact of event-related, pulsatile dopamine teaching signals. Based on this, we expected superior encoding for $9 \mathrm{R}$ compared to $10 \mathrm{R}$ homozygotes. We further predicted that this lower baseline dopamine and more optimized signal-to-noise ratio would render 9R-carriers more susceptible to disruption from exogenous dopamine therapy.

Consistent with our finding that PD patients with 9R-carrier status were more susceptible to dopamine overdose of VTAinnervated brain regions, Eisenegger et al. found that following L-DOPA treatment, healthy 9R-carriers were less able than their 10R homozygote counterparts to learn about the playing style of a partner in an interactive, competition-cooperation task (48). As in our study here with PD patients investigating learning of abstract images, in their study in healthy controls, dopaminergic therapy worsened 9R-carriers' ability to learn an adaptive strategy to maximize their winnings (48).

\section{Interpretation of Memory Retrieval Results}

Explicit retrieval processes implicate more distributed brain regions compared to encoding. Some of the brain regions implicated in retrieval overlap with encoding, such as hippocampus $(7,49)$, but the DS and cortical regions to which DS is reciprocally connected are also involved. In patients with DS lesions, explicit memory is commonly impaired $(50,51)$. Frontal lobe lesions, particularly in dorsolateral prefrontal cortex, which is an important cortical partner of DS, also commonly impair free recall $(52,53)$. Further, regions such as the dorsal frontoparietal network are engaged preferentially during free recall (49).

DS is seriously dopamine restricted in PD at baseline, even at early stages of disease. Functions performed by DS and its cortical partners are consistently improved by dopaminergic supplementation $(3,11)$. In the current study we replicated the finding that PD patients' recall ability is improved with the administration of dopaminergic therapy (11). We also found that for retrieval, unlike encoding, the effect of dopaminergic therapy was not modulated by genotype.

\section{SLC6A3 Effects on Recall On and Off Dopaminergic Therapy in PD}

The predictions regarding the effect of SLCA3 gene on recall were less clear than they were for encoding. Although more optimized signal-to-noise ratio in 9R-carriers might be expected to benefit recall performance, features of DS DAT concentration and dopamine regulation, as well as previous findings, made the predicted effects of SCLA3 gene on recall performance more complex. The cytoarchitectonics of DS are substantially different from those of VS. The high density of dopamine inputs on DS MSNs cause rapid, maximal responses through a wide range of firing frequencies and intensities (54, 55). Further, DS contains extremely high concentrations of DAT, resulting in short periods of dopaminergic stimulation (54). Therefore, dopaminergic stimulation in DS seems to produce a binary signal because brief and maximal responding occurs over very wide ranges of stimulation. Based on this, it seemed unlikely that subtle differences in SLC6A3 expression and small variations in signal-to-noise ratio related to SLC6A3 gene polymorphisms would significantly impact DS function.

Finally, we also expected that the effect of SLC6A3 genotype would be relatively negligible in the face of the substantial dopamine deficiency to DS that occurs at all stages of disease in PD. Motor and cognitive functions mediated by DS and its cortical partners are markedly impaired in the off state and are improved with dopaminergic therapy $(2-4,17)$. Consequently, we expected PD patients would recall more items on relative to off medication irrespective of SLC6A3 genotype. This prediction was borne out.

\section{CONCLUSION}

To our knowledge, this is the first investigation of the effect of SLC6A3 genotype on (a) cognitive functions mediated by VTA-innervated brain regions vs. DS, and (b) response to dopaminergic therapy in PD. Replicating our previous findings (11), dopaminergic therapy differentially affected explicit memory encoding and retrieval in PD patients. Dopaminergic therapy improved recall performance in $\mathrm{PD}$ patients irrespective of SLC6A3 genotype, whereas it impaired encoding, but only for 9R-carriers. This pattern of findings is in keeping with the view that encoding is mediated by dopamine-replete VTA-innervated brain regions, such as hippocampus and VS, whereas recall is mediated by broad brain regions including the significantly dopamine-deplete DS and its cortical partners. These results indicate that whereas dopaminergic therapy benefits DS function in all PD patients irrespective of SLC6A3 genotype, 9R-carrier status predisposes to dopamine overdose of VTA-innervated brain regions. We speculate that higher SLC6A3 expression in $9 \mathrm{R}$ allele carriers, and consequently lower basal dopamine, yields a more optimized signal-to-noise dopamine ratio that is more vulnerable to disruption by exogenous dopamine. This is in comparison to $10 \mathrm{R}$ homozygotes who are adapted to higher and more variable baseline, tonic dopamine. These results suggest that $9 \mathrm{R}$-carrier status predisposes to dopamine overdose.

\section{AUTHOR CONTRIBUTIONS}

PM, RK, KS, BR, and NH designed the experiment. BR, AA, AM, $\mathrm{NH}, \mathrm{US}, \mathrm{RK}$, and RT acquired and analyzed data. BR and PM wrote the manuscript. All authors edited the manuscript.

\section{FUNDING}

This work was supported by a Natural Sciences and Engineering Research Council of Canada grant (NSERC; Grant: RA4981A01), a Lawson Internal Research Fund Award (Lawson IRF), and a Canada Research Chair Tier 2 (CRC; Grant: 950-230372) to PM, and an Ontario Graduate Scholarship awarded to BR.

\section{ACKNOWLEDGMENTS}

We thank Mikayla Lynds and Emma MacDonald for assisting with data entry. 


\section{REFERENCES}

1. Kish SJ, Shannak K, Hornykiewicz O. Uneven pattern of dopamine loss in the striatum of patients with idiopathic parkinson's disease. N Engl J Med. (1988) 318:876-80. doi: 10.1056/NEJM198804073181402

2. MacDonald PA, Monchi O. Differential effects of dopaminergic therapies on dorsal and ventral striatum in parkinson's disease: implications for cognitive function. Park Dis. (2011) 2011:1-18. doi: 10.4061/2011/572743

3. Cools R. Dopaminergic modulation of cognitive function-implications for 1-DOPA treatment in Parkinson's disease. Neurosci Biobehav Rev. (2006) 30:1-23. doi: 10.1016/j.neubiorev.2005.03.024

4. Cools R, D’Esposito M. Inverted-U-shaped dopamine actions on human working memory and cognitive control. Biol. Psychiatry (2011) 69:e113-25. doi: 10.1016/j.biopsych.2011.03.028

5. Frank MJ. Dynamic dopamine modulation in the basal ganglia: a neurocomputational account of cognitive deficits in medicated and nonmedicated Parkinsonism. J Cogn Neurosci. (2005) 17:51-72. doi: 10.1162/0898929052880093

6. Lisman JE, Grace AA. The hippocampal-VTA loop: controlling the entry of information into long-term memory. Neuron (2005) 46:703-13. doi: 10.1016/j.neuron.2005.05.002

7. Spaniol J, Davidson PSR, Kim ASN, Han H, Moscovitch M, Grady CL. Event-related fMRI studies of episodic encoding and retrieval: meta-analyses using activation likelihood estimation. Neuropsychologia (2009) 47:1765-79. doi: 10.1016/j.neuropsychologia.2009.02.028

8. Petrides M. The Orbitofrontal cortex: novelty, deviation from expectation, and memory. Ann N Y Acad Sci. (2007) 1121:33-53. doi: 10.1196/annals.1401.035

9. Hampshire A, Hellyer PJ, Parkin B, Hiebert N, MacDonald P, Owen AM, et al. Network mechanisms of intentional learning. NeuroImage (2016) 127:123-34. doi: 10.1016/j.neuroimage.2015.11.060

10. Grogan J, Bogacz R, Tsivos D, Whone A, Coulthard E. Dopamine and consolidation of episodic memory: timing is everything. J Cogn Neurosci. (2015) 27:2035-50. doi: 10.1162/jocn_a_00840

11. MacDonald AA, Seergobin KN, Owen AM, Tamjeedi R, Monchi O, Ganjavi $\mathrm{H}$, et al. Differential effects of parkinson's disease and dopamine replacement on memory encoding and retrieval. PLoS ONE (2013) 8:e74044. doi: 10.1371/journal.pone.0074044

12. Hiebert NM, Seergobin KN, Andrew Vo, Hooman Ganjavi, MacDonald PA. Dopaminergic therapy affects learning and impulsivity in Parkinson's disease. Ann Clin Transl Neurol. (2014) 1:833-43. doi: 10.1002/acn3.128

13. Vo A, Hiebert NM, Seergobin KN, Stephanie Solcz, Allison Partridge, MacDonald PA. Dopaminergic medication impairs feedback-based stimulusresponse learning but not response selection in Parkinson's disease [Internet]. Front Hum Neurosci. (2014) 8:784. doi: 10.3389/fnhum.2014.00784

14. Gallant H, Vo A, Seergobin KN, MacDonald PA. Pramipexole Impairs Stimulus-Response Learning in Healthy Young Adults [Internet]. Front Neurosci. (2016) 10:374. doi: 10.3389/fnins.2016.00374

15. Vo A, Seergobin KN, Morrow SA, MacDonald PA. Levodopa impairs probabilistic reversal learning in healthy young adults. Psychopharmacology (2016) 233:2753-63. doi: 10.1007/s00213-016-4322-x

16. Cools R, Barker RA, Sahakian BJ, Robbins TW. 1-Dopa medication remediates cognitive inflexibility, but increases impulsivity in patients with Parkinson's disease. Neuropsychologia (2003) 41:1431-41. doi: 10.1016/S0028-3932(03)00117-9.

17. MacDonald P A, MacDonald AA, Seergobin KN, Tamjeedi R, Ganjavi H, Provost JS, et al. The effect of dopamine therapy on ventral and dorsal striatum-mediated cognition in Parkinson's disease: support from functional MRI. Brain J Neurol. (2011) 134(Pt 5):1447-63. doi: 10.1093/brain/awr075

18. MacDonald AA, Seergobin KN, Tamjeedi R, Owen AM, Provost JS, Monchi O, et al. Examining dorsal striatum in cognitive effort using Parkinson's disease and fMRI. Ann Clin Transl Neurol. (2014) 1:390-400. doi: 10.1002/acn3.62

19. Ciliax BJ, Drash GW, Staley JK, Haber S, Mobley CJ, Miller GW, et al. Immunocytochemical localization of the dopamine transporter in human brain. J Comp Neurol. (1999) 409:38-56. doi: 10.1002/(SICI)10969861(19990621)409:1<38::AID-CNE4>3.0.CO;2-1

20. Sano A, Kondoh K, Kakimoto Y, Kondo I. A 40-nucleotide repeat polymorphism in the human dopamine transporter gene. Hum Genet. (1993) 91:405-6. doi: 10.1007/BF00217369
21. Costa A, Riedel M, Müller U, Möller HJ, Ettinger U. Relationship between SLC6A3 genotype and striatal dopamine transporter availability: a metaanalysis of human single photon emission computed tomography studies. Synapse (2011) 65:998-1005. doi: 10.1002/syn.20927

22. Faraone SV, Spencer TJ, Madras BK, Zhang-James Y1, Biederman J. Functional effects of dopamine transporter gene genotypes on in vivo dopamine transporter functioning: a meta-analysis. Mol Psychiatry (2014) 19:880-89. doi: 10.1038/mp.2013.126

23. Forbes EE, Brown SM, Kimak M, Ferrell RE, Manuck SB, Hariri AR. Genetic variation in components of dopamine neurotransmission impacts ventral striatal reactivity associated with impulsivity. Mol Psychiatry (2009) 14:60-70. doi: 10.1038/sj.mp.4002086

24. Hariri AR, Brown SM, Williamson DE, Flory JD, de Wit H, Manuck SB. Preference for immediate over delayed rewards is associated with magnitude of ventral striatal activity. J Neurosci. (2006) 26:13213-17. doi: 10.1523/JNEUROSCI.3446-06.2006

25. Dreher J-C, Kohn P, Kolachana B, Daniel R. Weinberger, Berman KF. Variation in dopamine genes influences responsivity of the human reward system. Proc Natl Acad Sci USA. (2009) 106:617-22. doi: 10.1073/pnas.0805517106

26. Brody AL, Mandelkern MA, Olmstead RE, Scheibal D, Hahn E, Shiraga S, et al. Gene variants of brain dopamine pathways and smoking-induced dopamine release in the ventral caudate/nucleus accumbens. Arch Gen Psychiatry (2006) 63:808-16. doi: 10.1001/archpsyc.63.7.808

27. Garcia-Garcia M, Barceló F, Clemente IC, Escera C. The role of the dopamine transporter DAT1 genotype on the neural correlates of cognitive flexibility. Eur J Neurosci. (2010) 31:754-60. doi: 10.1111/j.1460-9568.2010.07102.x

28. Habak C, Noreau A, Nagano-Saito A, Mejía-Constaín B, Degroot C, Strafella AP, et al. Dopamine transporter SLC6A3 genotype affects cortico-striatal activity of set-shifts in Parkinson's disease. [Internet]. Brain J Neurol. (2014) 137:3025-35. doi: 10.1093/brain/awu251

29. Schott BH. The Dopaminergic midbrain participates in human episodic memory formation: evidence from genetic imaging. J Neurosci. (2006) 26:1407-17. doi: 10.1523/JNEUROSCI.3463-05.2006

30. Bertolino A, Fazio L, Di Giorgio A, Blasi G, Romano R, Taurisano P, et al. Genetically determined interaction between the dopamine transporter and the $\mathrm{d} 2$ receptor on prefronto-striatal activity and volume in humans. J Neurosci. (2009) 29:1224-34. doi: 10.1523/JNEUROSCI.4858-08.2009

31. Eisenegger C, Naef M, Linssen A, Clark L, Gandamaneni PK, Müller U, et al. Role of dopamine D2 receptors in human reinforcement learning. Neuropsychopharmacology (2014) 39:2366-75. doi: 10.1038/npp.2014.84

32. Robertson BD, Hiebert NM, Seergobin KN, Owen AM, MacDonald PA. Dorsal striatum mediates cognitive control, not cognitive effort per se, in decision-making: an event-related fMRI study. NeuroImage (2015) 114:17084. doi: 10.1016/j.neuroimage.2015.03.082

33. Shrager Y, Kirwan CB, Squire LR. Activity in both hippocampus and perirhinal cortex predicts the memory strength of subsequently remembered information. Neuron (2008) 59:547-53. doi: 10.1016/j.neuron.2008.07.022

34. Evans AH, Katzenschlager R, Paviour D, O'Sullivan JD, Appel S, Lawrence AD, et al. Punding in Parkinson's disease: its relation to the dopamine dysregulation syndrome. Mov Disord. (2004) 19:397-405. doi: $10.1002 / \mathrm{mds} .20045$

35. Cook EH Jr, Stein MA, Krasowski MD, Cox NJ, Olkon DM, Kieffer JE, et al. Association of attention-deficit disorder and the dopamine transporter gene. Am J Hum Genet. (1995) 56:993-8.

36. Vandenbergh DJ, Persico AM, Hawkins AL, Griffin CA, Li X, Jabs EW, et al. Human dopamine transporter gene (DAT1) maps to chromosome 5p15. 3 and displays a VNTR. Genomics (1992) 14:1104-06. doi: 10.1016/S0888-7543(05)80138-7

37. Majdan A, Sziklas V, Jones-Gotman M. Performance of healthy subjects and patients with resection from the anterior temporal lobe on matched tests of verbal and visuoperceptual learning. J Clin Exp Neuropsychol. (1996) 18:416-30. doi: 10.1080/016886396084 08998

38. Vakil E, Raz T, Levy DA. Probing the brain substrates of cognitive processes responsible for context effects on recognition memory. Aging Neuropsychol Cogn. (2010) 17:519-44. doi: 10.1080/13825581003690182

39. Wixted JT, Ebbesen EB. On the form of forgetting. Psychol Sci. (1991) 2:40915. doi: 10.1111/j.1467-9280.1991.tb00175.x 
40. Schacht JP. COMT val158met moderation of dopaminergic drug effects on cognitive function: a critical review. Pharmacogenomics J. (2016) 16:430-38. doi: $10.1038 /$ tpj.2016.43

41. Hiebert NM, Vo A, Hampshire A, Owen AM, Seergobin KN, MacDonald PA. Striatum in stimulus-response learning via feedback and in decision making. NeuroImage (2014) 101:448-57. doi: 10.1016/j.neuroimage.2014. 07.013

42. Atallah HE, Lopez-Paniagua D, Rudy JW, O’Reilly RC. Separate neural substrates for skill learning and performance in the ventral and dorsal striatum. Nat Neurosci. (2007) 10:126-31. doi: 10.1038/nn1817

43. Bellebaum C, Koch B, Schwarz M, Daum I. Focal basal ganglia lesions are associated with impairments in reward-based reversal learning. Brain (2008) 131:829-41. doi: 10.1093/brain/awn011

44. Reiss JP, Campbell DW, Leslie WD, Paulus MP, Stroman PW, Polimeni JO, et al. The role of the striatum in implicit learning: a functional magnetic resonance imaging study. Neuroreport (2005) 16:1291-95. doi: 10.1097/01.wnr.0000175615.93312.1a

45. Shohamy D, Myers CE, Geghman KD, Sage J, Gluck MA. 1-dopa impairs learning, but spares generalization, in Parkinson's disease. Neuropsychologia (2006) 44:774-84. doi: 10.1016/j.neuropsychologia.2005.07.013

46. Chiaravalloti ND, Ibarretxe-Bilbao N, DeLuca J, Rusu O, Pena J, GarcíaGorostiaga I, et al. The source of the memory impairment in Parkinson's disease: acquisition versus retrieval: memory in parkinson's disease. Mov Disord. (2014) 29:765-71. doi: 10.1002/mds.25842

47. Ellfolk U, Joutsa J, Rinne JO, Parkkola R, Jokinen P, Karrasch M. Brain volumetric correlates of memory in early Parkinson's disease. J Park Dis. (2013) 3:593-601. doi: 10.3233/JPD-130276

48. Eisenegger C, Pedroni A, Rieskamp J, Zehnder C, Ebstein R, Fehr E, et al. DAT1 polymorphism determines L-DOPA effects on learning about others' prosociality. PLoS ONE (2013) 8:e67820. doi: 10.1371/journal.pone.0067820

49. Kragel JE, Polyn SM. Decoding episodic retrieval processes: frontoparietal and medial temporal lobe contributions to free recall. J Cogn Neurosci. (2016) 28:125-39. doi: 10.1162/jocn_a_00881
50. Benke T, Delazer M, Bartha L, Auer A. Basal ganglia lesions and the theory of fronto-subcortical loops: neuropsychological findings in two patients with left caudate lesions. Neurocase (2003) 9:70-85. doi: 10.1076/neur.9.1.70.14374

51. Su C-Y, Chen H-M, Kwan A-L, Lin YH, Guo NW. Neuropsychological impairment after hemorrhagic stroke in basal ganglia. Arch Clin Neuropsychol. (2007) 22:465-74. doi: 10.1016/j.acn.2007.01.025

52. Gershberg FB, Shimamura AP. Impaired use of organizational strategies in free recall following frontal lobe damage. Neuropsychologia (1995) 33:1305-33.

53. Stuss, DT, Alexander MP, Palumbo CL, Buckle L, Sayer L, Pogue J. Organizational strategies with unilateral or bilateral frontal lobe injury in word learning tasks. Neuropsychology (1994) 8:355. doi: 10.1037/0894-4105.8.3.355

54. Wickens JR, Budd CS, Hyland BI, Arbuthnott GW. Striatal contributions to reward and decision making: making sense of regional variations in a reiterated processing matrix. Ann N Y Acad Sci. (2007) 1104:192-212. doi: 10.1196/annals.1390.016

55. Zhang T, Zhang L, Liang Y, Athanassios G. Siapas, Zhou F, Dani JA, et al. Dopamine signaling differences in the nucleus accumbens and dorsal striatum exploited by nicotine. J. Neurosci. (2009) 29:4035-43. doi: 10.1523/JNEUROSCI.0261-09.2009

Conflict of Interest Statement: The authors declare that the research was conducted in the absence of any commercial or financial relationships that could be construed as a potential conflict of interest.

Copyright (c) 2018 Robertson, Al Jaja, MacDonald, Hiebert, Tamjeedi, Seergobin, Schwarz, Kim and MacDonald. This is an open-access article distributed under the terms of the Creative Commons Attribution License (CC BY). The use, distribution or reproduction in other forums is permitted, provided the original author(s) and the copyright owner(s) are credited and that the original publication in this journal is cited, in accordance with accepted academic practice. No use, distribution or reproduction is permitted which does not comply with these terms. 